\title{
Humoral Immune Response of Inactivated Bivalent Leptospira Vaccine among Dogs in Tiruchirappalli, Tamilnadu, India*
}

\author{
Kalimuthusamy Natarajaseenivasan ${ }^{1 \#}$, Chandramohan Lakshmi Priya ${ }^{1}$, Shanmugam Vanithamani ${ }^{1}$, \\ Santhanam Shanmughapriya ${ }^{1}$, Subbiah Anandhagiri ${ }^{2}$ \\ ${ }^{1}$ Medical Microbiology Laboratory, Department of Microbiology, School of Life Sciences, Bharathidasan University, Tiruchirappalli, \\ India; ${ }^{2}$ Department of Animal Husbandry, Government Veterinary Clinic, Tiruchirappalli, India. \\ Email: "natarajaseenivasan@rediffmail.com
}

Received October $10^{\text {th }}, 2011$; revised December $23^{\text {rd }}, 2011$; accepted January $28^{\text {th }}, 2012$

\begin{abstract}
Leptospirosis has been recognized as the disease of the dogs. The prevention of canine leptospirosis can block the transmission of the etiological agent to humans. Commercial vaccines prevent not only clinical leptospirosis but also the renal carrier state in dogs. Thus in this present investigation, the humoral immune response of the vaccinated dogs with the multivalent vaccine (Megavac-6) was analyzed. Enzyme linked immunosorbent assay (ELISA) with preparations of 2 whole cell lysate, 2 leptospiral LPS, 4 purified recombinant proteins were used to test sera from 30 dogs vaccinated with MEGAVAC-6, a commercial vaccine practiced by the animal husbandary Departments in Tamilnadu. All 30 sera were positive by ELISA with whole cell lysate of Canicola and Icterohaemorrhagiae, and ELISA with Canicola LPS, Icterohaemorrhagiae LPS; antibody titres ranged from 20 to $\geq 10,240$. Although there was less frequent reactivity of sera with recombinant antigens, antibodies to LipL32 and LigA were detected in 25 (83.3\%) serum samples. Less frequent reactivity was noted when recombinant GroEl, LK73.5 antigens were included separately in ELISAs. The highest MAT titre was observed against the serovar Canicola and Icterohaemorrhagiae. The more reactivity against LPS may be due to the dominance of serovar specific leptospiral LPS, which may be the dominant immunogenic antigen in inactivated bivalent leptospiral vaccines. Eventhough LPS is the dominant antigen. It is serovars specific and hence recommends the incorporation of the locally circulating serovars in vaccine for its efficient use. The efficiency at this point can be increased by the use of subunit vaccines rather than recombinant proteins as such. The present study has proposed certain epitopes with increased antigenic potency.
\end{abstract}

Keywords: Recombinant Proteins; LPS; Humoral Response; Canine Vaccine

\section{Introduction}

Leptospirosis is one of the most common bacterial zoonoses worldwide [1]. It occurs most frequently in developing countries of tropical and sub tropical regions that have relatively poor sanitation and waste disposal practices [2] (WHO 2003). Humans and animals become infected when they are directly exposed to pathogenic Leptospira from other infected animals or indirectly by contact with soil or water that has been contaminated with the urine of animals shedding the organism. There are several animal reservoirs that allow the organisms to be maintained in the environment [3-6]. Historically leptospirosis was recognized as a disease of dogs before it

\footnotetext{
${ }^{*}$ Competing interests: The authors declare that they have no conflict of interest.

${ }^{\#}$ Corresponding author.
}

was known in any other animal species, including humans [7]. L. interrogans Canicola and Icterohaemorrhagiae were considered as the most significant serovars for canine leptospirosis worldwide [8]. Although acute leptospirosis may result in death of the dog, those that survive may harbor the leptospires in their kidneys, becoming persistent shedders of the leptospires in urine. Infection of humans with Canicola and Icterohaemorrhagiae have been connected with the coexistence and close contact between dogs and humans resulting in the transmission of the etiological agent among veterinarians, dog breeders and dog owners. Several severe epidemics of human leptospirosis have been reported most likely due to exposure to Canicola infected dogs' urine contaminated flood waters [9].

In Europe and North America the prevalence of the serovar Canicola and to lesser extent serovar Ictero- 
haemorrhagiae infection in dogs is believed to have decreased because of the wide spread use of canine vaccines against these two serovars [10]. Most commercial vaccines against canine leptospirosis are chemically inactivated whole cell vaccines. In the 1980s and 1990s the efficacy of the available commercial vaccines in preventing not only clinical leptospirosis but also the renal carrier state in dogs and other animal species was extensively discussed, since in some this efficacy was questioned $[11,12]$.

Thus in this present investigation the humoral immune response of the vaccinated dogs with the multivalent vaccine (Megavac-6) was analyzed. The humoral immune response of the vaccinated dogs against the leptospiral whole cell lysate, lipopolysaccharide (LPS), recombinant LipL32, GroEL, LK73.5 and LigA was assessed in different time intervals.

\section{Methods}

\subsection{Animals}

Blood samples were collected from dogs attending the Government Veterinary Hospital, Department of Animal Husbandry, Palakari. Dogs of both sex, different breeds and age were chosen from the dogs brought to the hospital from in and around Tiruchirapalli district, Tamilnadu. The samples were screened for the presence of specific antibodies against Leptospira with serological assays (ELISA and MAT) prior to immunization (sample number: DVI). Only animal negative for leptospiral antibodies were included in the study. Such animals were assigned for vaccination. At the time of vaccination all dogs were healthy, based on pulse, temperature and clinical examination. This study was approved by the Department of animal husbandry, Chennai, Tamil Nadu with the vide reference number $77721 / \mathrm{JJ} / 09$ dated 2.12.2009.

\subsection{Commercial Vaccines}

MEGAVAC-6 is the commercial vaccine currently supplied by the Government of Tamilnadu for vaccinating the dogs. It is a multivalent vaccine containing freeze dried form of canine distemper, canine contagious hepatitis and canine parvo virus. Apart from this the aqueous vaccine also consists of inactivated Leptospira serovars Canicola and Icterohaemorrhagiae.

\subsection{Vaccination and Serology}

Vaccines were administered subcutaneously either at the chest wall or between the shoulders. Based on the serological analysis (i.e., negative by MAT) 30 animals were selected for receiving vaccination. All the dogs were vaccinated twice with 3 weeks interval. Beginning with the second vaccination (DV-II), blood samples of all the dogs participating in the study were collected after each immunization. The last sample (DV-IV) was taken after the booster vaccination administered $(375 \pm 3)$ days after the first immunization. Blood was drawn either by cephalic or sephaneous vein puncture as per the physicians' direction. The collected blood samples were allowed to coagulate centrifuged at $3500 \times \mathrm{g}$ for $15 \mathrm{mins}$ and the resulting sera were stored at $-20^{\circ} \mathrm{C}$.

\subsection{Antigen Preparation}

L. interrogans serovar Canicola and Icterohaemorrhagiae were grown in EMJH medium to the desired density of 1 $-2 \times 10^{8}$ organisms $/ \mathrm{ml}$. The culture thus obtained was centrifuged at $10,000 \times \mathrm{g}$ for 10 mins. The supernatant was discarded and pellet resuspended and washed twice with sterile PBS ( $\mathrm{pH}$ 7.4). Ultrasound disruption of $L$. interrogans was performed on ice at $35 \mathrm{~W}$ for $10 \mathrm{~s}$ repeating it for 10 mins. The sonicated preparation was centrifuged at $10,000 \times \mathrm{g}$ at $4^{\circ} \mathrm{C}$ for $10 \mathrm{~min}$. The supernatant thus obtained was subjected for acetone precipitation. The protein concentration was measured using BCA protocol.

Leptospiral LPS was extracted by phenol-water extraction (PW) method as described earlier [13]. Lyophilized leptospiral cultures ( $0.15 \mathrm{mg}$ dry weight) were suspended in $100 \mathrm{ml}$ of water at $68^{\circ} \mathrm{C}$, mixed with an equal volume of $90 \%(\mathrm{wt} / \mathrm{vol})$ phenol-water, and shaken for 15 min, cooled in an ice bath, centrifuged at $3000 \mathrm{xg}$ for 10 min, and the aqueous layer recovered. The phenol layer was re-extracted with water twice. The aqueous layers were collected, dialyzed against running water, centrifuged at $105,000 \times \mathrm{g}$ for $1 \mathrm{~h}$, and the LPS pellet was lyophilized and dissolved in MQ and estimated as per Duboi's method (1956) [14]. The recombinant LipL32 and GroEL were produced in our laboratory and procedure described elsewhere. Recombinant proteins including LigA and LK73.5 utilized in this study was the kind gift of Prof. John F. Timoney, University of Kentucky, USA.

\subsection{Serological Analysis}

The microscopic agglutination test (MAT) was performed using 13 leptospiral reference serovars-Australis (JezBratislava), Autumnalis (Bankinang), Ballum (Mus127), Bataviae (VanTienen), Canicola (HondUtrechIV), Grippotyphosa (MoskvaV), Hebdomadis (Hebdomadis), Icterohaemorrhagiae (RGA), Javanica (VeldratBataviae46), Sejroe (Hardjoprajitno), Pomona (Pomona), Pyrogenes (Salinem), and Tarassovi (Perepilitsin). All these serovars were received from the Leptospira WHO Reference Centre, Port Blair and maintained with periodic subculture 
in EMJH medium at the Medical Microbiology Laboratory, Department of Microbiology, Bharathidasan University, Tiruchirappalli. The MAT was performed at doubling dilutions starting from 1:20. Seven-day-old cultures at a concentration of $1-2 \times 10^{8}$ organisms $/ \mathrm{ml}$ were used as antigen. Positive cut-off values for dogs were based on values of the previous studies $[15,16]$.

\subsection{Enzyme Linked Immunosorbent Assay (ELISA)}

IgG ELISA was done following standard procedure [17] to assess the humoral immune response of the vaccinated dogs against various Leptospira antigens including sonicated whole cell lysate of Leptospira interrogans serovar Canicola, Icterohaemorrahagiae, recombinant proteins LipL32, GroEL, LigA, LK73.5 and leptospiral LPS. Antigens were coated in each well $(1 \mu \mathrm{g} / \mathrm{well})$ of the flatbottomed polystyrene microtitre plates and they were kept overnight at $4^{\circ} \mathrm{C}$. The antigen coated plates were rinsed three times with PBS/Tween 20 (PBST). Then blocked with $4 \%$ non-fat milk and incubated at $37^{\circ} \mathrm{C}$ for 1 hour. After washing, the serum samples were diluted in doubling dilutions starting from 1:10 and the plates were incubated for $1 \mathrm{hr}$ at $37^{\circ} \mathrm{C}$. After incubation the plates were washed with PBST and incubated with $100 \mu$ of conjugate in 1:4000 dilutions (Anti-Dog IgG-HRP conjugate, Sigma) at $37^{\circ} \mathrm{C}$ for $1 \mathrm{~h}$. Finally $100 \mu \mathrm{l}$ of Ortho Phenelene Diamine (OPD) substrate was added to all the wells and the reaction was stopped by adding $50 \mu 1$ of 2 $\mathrm{N}$ sulfuric acid. The OD was read at $490 \mathrm{~nm}$.

\subsection{Prediction of B-Cell Epitope}

Protein sequences of LipL32, LigA, LK73.5 and GroEL of $L$. interrogans were obtained from NCBI and were subjected for BCPreds [18]. BCPred identifies common B-cell epitopes. The epitopes with BCPreds score of $>0.8$ and VaxiJen score $>0.4$ were predicted as highly immunogenic epitope that can be used for the preparation of subunit vaccines.

\section{Results}

\subsection{Animals}

Of the 74 dogs analyzed, 17 (23\%) reacted positive for $L$. interrogans antibodies by MAT. The infection rate was $16.2 \%$ (12 animals) and the remaining 5 animals (6.8\%) had vaccine induced antibodies. Seropositivity was not accompanied by any clinical signs of illness. 57 of 74 dogs tested were chosen for vaccination study. The 57 dogs were divided into 2 groups: Group A $(\mathrm{n}=30)$ received vaccine as per the vaccination schedule; Group B $(\mathrm{n}=27)$ served as seronegative controls and not administered with vaccine.

\subsection{Serological Analysis of the Control Animals}

Using the control animals a MAT titre of 1 in 20 was considered as the cut-off titre. Following vaccination, the MAT titre raised above the cut-off titre confirming an induction of the humoral immune response by the vaccine (Figure 1). As expected the highest titre was observed against the serovar Canicola and Icterohaemorrhagiae.

In ELISA, the cut off OD (Mean + 2SD) for Canicola sonicated antigen, Icterohaemorrhagiae sonicated antigen, Canicola LPS, Icterohaemorrhagiae LPS, rLipL32, rGroEL, rLigA, rLK73.5 was calculated and found to be 0.147 , $0.131,0.134,0.126,0.132,0.125,0.130$ and 0.129 respectively. The samples having titres greater than the cut off OD were considered positive for the presence of leptospiral antibodies (Figure 2).

\subsection{Serological Analysis of Vaccinated Dogs}

Thirty sera from vaccinated dogs were analyzed separately with whole cells or recombinant antigens. All 30 sera were positive by ELISA with whole cell lysate of Canicola and Icterohaemorrhagiae, and ELISA with Canicola LPS, Icterohaemorrhagiae LPS; antibody titres ranged from 20 to $\geq 10,240$. Although there was less frequent reactivity of sera with recombinant antigens (Table 1), antibodies to LipL32 and LigA were detected in 25 $(83.3 \%)$ cases. Less frequent reactivity was noted when recombinant GroEl, LK73.5 antigens were included separately in ELISAs. In general the proportions of positive sera were highest in ELISAs that contained the whole cell lysate of Canicola and Icterohaemorrhagiae followed by Canicola LPS, Icterohaemorrhagiae LPS, and recombinant antigens including LipL32 and LigA in separate analysis.

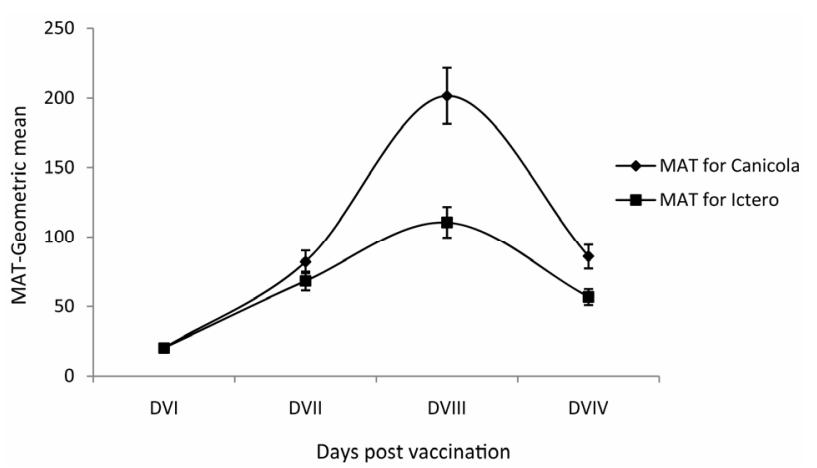

Figure 1. Humoral immune response of vaccinated dogs against Leptospira interrogans serovar Canicola and Icterohaemorrhagiae by microscopic agglutination test (MAT). X-axis-Days of post vaccination, DVI-0 day, DVII-21 days, DVIII-42 days, DVIV-365 days; Y-axis-Geometric mean antibody titre by MAT. 


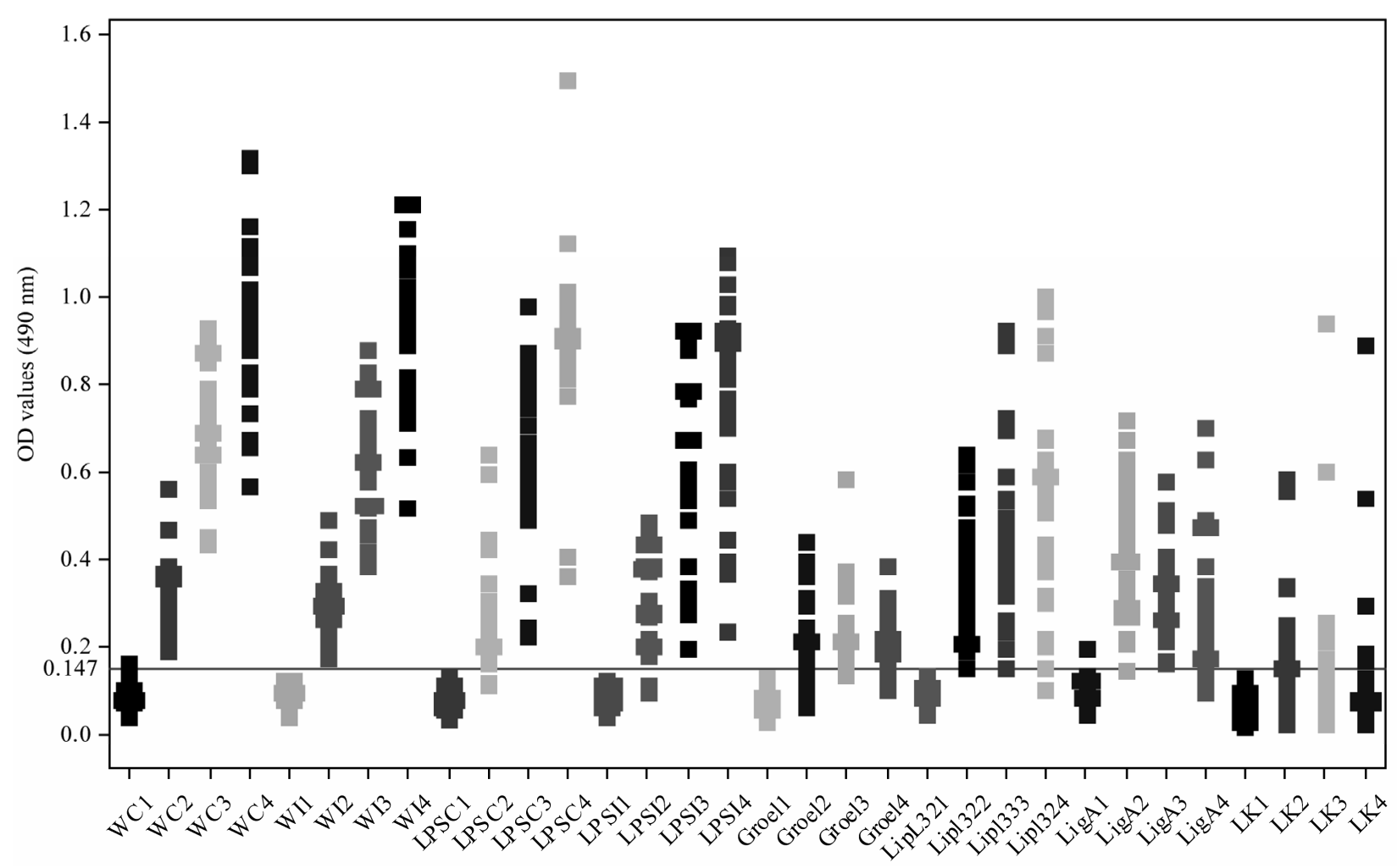

Figure 2. Humoral immune response of vaccinated dogs against various antigenic fractions of Leptospira by ELISA: X-axis: Different vaccination schedule and antigenic determinants; Y-axis: Optical density at $490 \mathrm{~nm}$.

Table 1. Particulars about the animals included to estimate the humoral immune response against the Megavac-6.

\begin{tabular}{|c|c|c|c|c|c|c|}
\hline \multirow{2}{*}{ Antigens tested } & \multicolumn{3}{|c|}{ ELISA results (Geometric mean of antibody titre) } & \multicolumn{3}{|c|}{ Number of seropositive cases } \\
\hline & DVII & DVIII & DVIV & DVII & DVIII & DVIV \\
\hline Whole cell (Canicola) & 108.03 & 970.06 & 3300.79 & 30 & 30 & 30 \\
\hline Whole cell (Icterohaemorrhagiae) & 103.15 & 1015.94 & 3620.39 & 30 & 30 & 30 \\
\hline LPS (Canicola) & 83.78 & 670.27 & 2177.71 & 30 & 30 & 30 \\
\hline LPS (Icterohaemorrhagiae) & 87.75 & 654.96 & 2127.97 & 30 & 30 & 30 \\
\hline LipL32 & 80 & 259.92 & 376.18 & 25 & 30 & 30 \\
\hline LigA & 76.39 & 248.18 & 359.19 & 25 & 30 & 30 \\
\hline GroEL & 43.87 & 236.98 & 305.55 & 4 & 30 & 30 \\
\hline LK 73.5 & 51.57 & 231.56 & 342.97 & 11 & 30 & 30 \\
\hline
\end{tabular}

Geometric mean titres were elevated when sera were screened in ELISAs with whole cell and LPS antigens (Figure 3). Maximal antibody titres $(\geq 10,240)$ were recorded when dog sera were tested with whole cell antigen of Canicola and Icterohaemorrhagiae.

\subsection{Prediction of B-Cell Epitope}

The sequence of all the proteins analysed by VaxiJen v2.0 server gave scores showing the proteins as antigenic in nature. The BCPreds predicted the epitopes for B-cell response. The epitopes with VaxiJen scores $>0.4$ were considered as antigenic. Finally 9 out of 16 epitopes from LK73.5, 2 out of 7 from LipL32, 2 out of 6 from LigA and 8 out of 14 from GroEL were predicted as epitopes of B-cell response (Table 2).

\section{Discussion}

Canine leptospirosis occurs worldwide and it is usually caused by $L$. interrogans serovar Canicola and Icterohaemorrhagiae. Canine leptospirosis surveillance is an 


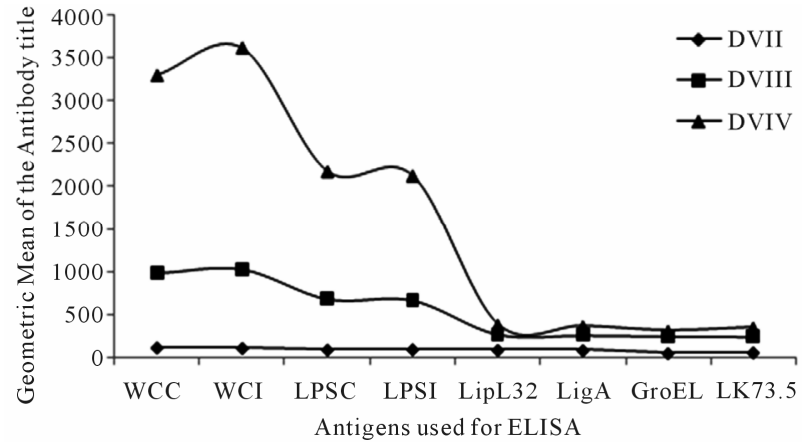

Figure 3. Humoral immune response of vaccinated dogs against various antigenic fractions of Leptospira by enzyme linked immunosorbent assay (ELISA). X-axis-Different antigenic determinants, DVI-0 day, DVII-21 days, DVIII-42 days, DVIV- 365 days; Y-axis-Geometric mean of the antibody titre.

Table 2. Selected B-cell epitopes using BCpreds and the antigenicity of peptides using VaxiJen.

\begin{tabular}{cclc}
\hline No & $\begin{array}{c}\text { Protein } \\
\text { (VaxiJen score) }\end{array}$ & \multicolumn{1}{c}{ Epitope sequence } & $\begin{array}{c}\text { VaxiJen } \\
\text { Score }\end{array}$ \\
\hline & & FLPNQTNSNQV & 1.0158 \\
& & NPASANS & 1.0093 \\
& & NYFTKEDSSNNIPKKVNSKN & 0.8355 \\
1 & LK73.5 (0.4813) $)$ & WGHDERAKRISK & 1.4741 \\
& & VKINKEGK & 0.9018 \\
& & THAQSQD & 2.3203 \\
& & YADPSTTKSGHK & 1.2601 \\
& & IQADPNRK & 1.6407 \\
2 & LipL32 (0.9409) & QKLDDDDDGDDTYKEERH & 1.5223 \\
& & YKPGEVK & 2.1960 \\
3 & LigA (0.8216) & VVIENTPGK & 1.3782 \\
& & TALSVGSSK & 1.1031 \\
& & VTLGPKG & 1.2767 \\
& & QMVKEVSTKT & 0.9879 \\
& & RAVKIEN & 1.9761 \\
4 & GroEL (0.5355) & LIYDKKI & 1.4475 \\
& & APGFGDRRK & 0.9526 \\
& & KQIEDTT & 1.0094 \\
& & TEVEMKEKK & 1.8835 \\
& & EHAKAKKGN & 2.5276 \\
\hline
\end{tabular}

important tool to detect the circulating serovars and it is very essential for the preparation of new vaccine candidates and also to avoid the risk of human exposure. The prevalence of leptospiral serovars significantly varied from country to country and the prevention of leptospirosis without vaccination is fairly difficult. Commercial Leptospira vaccines are available globally for cattle, pigs and dogs but vaccination will be effective only if the locally prevalent serovars were included in the preparation. Thus leptospiral animal vaccines are prepared using two or more locally prevalent serovars in their inactivated form. In this regard serovar Canicola and Icterohaemorrhagiae has been reported for canine and animal leptospirosis in Tamilnadu [16] and consequently Mega- vac- 6 , the commercially available vaccine contains the inactivated $L$. interrogans serovar Canicola and Icterohaemorrhagiae. Due to paucity of the data on the humoral immune response of Megavac vaccinated dogs and the efficiency of the vaccine, the present study is unique in its kind.

ELISA was carried out to demonstrate the immune response of vaccinated dogs using whole cell lysate, purified LPS and recombinant proteins as antigens. Twenty one days after primary vaccination a considerable level of immune response was observed and a maximum level of antibody was observed after 21 days from booster vaccination. Leptospiral whole cell lysates and LPS elicited a high level of antibody response compared to other antigenic preparations. The MAT results also envisaged a better humoral immune response with a high antibody titre of 1 in 10,240 for the serovar Canicola.

Such high level of antibody titre against whole cell lysates and LPS as detected by ELISA may be due to the presence of whole cell inactivated antigenic preparation of the leptospiral serovars Canicola and Icterohaemorrhagiae in the Megavac-6. Moreover leptospiral LPS act as a predominant antigen rather than any other proteins and hence the results indicate the protective efficiency of LPS. More studies are required to arrive for a firm confirmation.

Several studies have indicated LipL32 and LigA as immunodominant proteins; the low level of antibody titre against these proteins seems surprising. The possible reasons for such results may be that these proteins may excessively be responsible during in vivo infection of leptospires. Secondarily the low antigenic property of the whole protein as predicted from the VaxiJen score can thus be correlated to the slightly reduced reactivity of the proteins in ELISA. The highly antigenic epitopes would have been buried in the complex structure of proteins and thus unavailable for induction of immunogenic response and consequently reduced immune response in vaccinated dogs.

The results of the present study revealed a serovar specific nature. Further the DV-IV samples showed the declining phase of the antibody level for the various antigenic fractions indicating a need for annual revaccination to prevent the dogs from leptospirosis. Since the vaccines largely confer serovar-specific immunity, continuous epidemiological monitoring of the prevalent Leptospira serovars in a zone or region is desired to select the correct serovars for incorporating them in to vaccine [19]. Such underlying difficulties can be trounced over by the use of subunit vaccines designed with the predicted epitopes of the major leptospiral proteins. Such vaccine candidate can promise increased specificity and antigenic potency than that of attenuated live vaccines or recombinant pro- 
teins as whole.

\section{Authors' Contributions}

KNS designed the study, KNS and SS drafted the MS. CLP and SV carried out the experiments and SA collected the samples. All authors have read and approved the final manuscript.

\section{Acknowledgements}

We thank Department of Biotechnology, Government of India, Ministry of Science and Technology, New Delhi for funding (BT/PR13502/MED/15/36/2010). We thank Prof. JFT for the recombinant proteins. The authors are also thankful to the Veterinary Assistant Surgeons, Government Veterinary Clinic, Palakari, Tiruchirappalli for their help for the sample collection from the dogs. We also thank the Vice-Chancellor, Bharathidasan University and the Director Animal Husbandry for the facilities and permission provided to carry out this research work.

\section{REFERENCES}

[1] P. N. Levett, "Leptospirosis: A Forgotten Zoonosis," Clinical and Applied Immunology Reviews, Vol. 4, No. 6, 1994, pp. 435-448. doi:10.1016/j.cair.2004.08.001

[2] World Health Organisation, "Human Leptospirosis: Guidance for Diagnosis, Surveillance and Control 2003," WHO Library Cataloguing-in Publication Data, Geneva, 2003.

[3] N. B. Vanasco, M. D. Sequeira, G. Sequeira and H. D. Tarabla, "Associations between Leptospiral Infection and Seropositivity in Rodents and Environmental Characteristics in Argentina," Preventive Veterinary Medicine, Vol. 60, No. 3, 2003, pp. 227-235.

[4] T. Meeyam, P. Tablerk, B. Petchanok, D. Pichpol and P. Padungtod, "Seroprevalence and Risk Factors Associated with Leptospirosis in Dogs," Southeast Asian Journal of Tropical Medicine and Public Health, Vol. 37, No. 1, 2006, pp. 148-153.

[5] C. O. R. Everard, G. M. Fraser-Chanpong, L. J. Bhagwandin, M. W. Race and A. C. James, "Leptospires in Wildlife from Trinidad and Grenada," Journal of Wildlife Disease, Vol. 19, No. 3, 1983, pp. 192-199.

[6] C. O. R. Everard, G. M. Fraser-Chanpong, A. C. James and L. V. Butcher, "Serological Studies on Leptospirosis in Livestock and Chickens from Grenada and Trinidad," Transactions of the Royal Society of Tropical Medicine and Hygiene, Vol. 79, No. 6, 1985, pp. 859-864. doi:10.1016/0035-9203(85)90138-5

[7] S. Faine, "Leptospira and Leptospirosis," CRC Press, Boca Raton, 1994.
[8] H. L. B. M. Klaasen, M. J. C. H. Molkenboer, M. P. Vrijenhoek and M. J. Kaashoek, "Duration of Immunity in Dogs Vaccinated against Leptospirosis with a Bivalent Inactivated Vaccine," Veterinary Microbiology, Vol. 95, No. 1-2, 2003, pp. 121-132. doi:10.1016/S0378-1135(03)00152-4

[9] R. T. Trevejo, J. G. Rigau-Perez, D. A. Ashford, E. M. McClure, C. Jarquin-Gonzales, J. J. Amador, J. O. De los Reyes, A. Gonzales, S. R. Zaki, W. J. Shieh, R. G. McLean, R. S. Nasci, R. S. Weyant, C. A. Bolin, S. L. Bragg, B. A. Perkins and R. A. Spiegel, "Epidemic Leptospirosis Associated with Pulmonary Hemorrhage Nicaragua, 1995," Journal of Infectious Diseases, Vol. 178, 1998, No. 5, pp. 1457-1463. doi:10.1086/314424

[10] I. Ferguson, "A European Perspective on Leptospirosis," Microbiology Europe, 1994, pp. 8-11.

[11] V. Marshall, "Efficacy of Leptospira Vaccine," Journal of American Veterinary Medical Association, Vol. 183, No. 1, 1983, pp. 33-34.

[12] J. S. Wohl, "Canine leptospirosis," Compendium and Veterinary Technician, Vol. 18, No. 11, 1996, pp. 1215-1241.

[13] J. N. Saddler and A. C. Wardlaw, "Extraction, Distribution and Biodegradation of Bacterial Lipopolysaccharides in Estuarine Sediments," Antonie van Leeuwenhoek, Vol. 46, No. 1, 1980, pp. 27-39. doi:10.1007/BF00422226

[14] M. Dubois, K. A. Gilles, J. K. Hamilton, P. Rebers and F. Smith, "Colorimetirc Methods for the Determination of Sugars and Related Substances," Annals of Chemistry, Vol. 28, No. 3, 1956, pp. 350-356. doi: $10.1021 / \mathrm{ac} 60111 \mathrm{a} 017$

[15] K. Natarajaseenivasan and S. Ratnam, "Seroprevalence of Leptospiral Infection in an Agricultural Based Village in Tamilnadu," Cheiron, Vol. 26, No. 5-6, 1997, pp. 80-83.

[16] S. Ratnam, S. Subramanian and T. Sundararaj, "Evidence of Leptospiral Antibodies among Domestic Animals in Madras City," Cheiron, Vol. 12, 1983, No. 4, pp. 176179.

[17] W. J. Terpstea, G. S. Lighthart and C. J. Schoone, "ELISA for the Detection of Specific IgM and IgG in Human Leptospirosis," Journal of General Microbiology, Vol. 131, No. 2, 1985, pp. 377-385.

[18] Y. El-Manzalawy, D. Dobbs and V. Honavar, "Predicted Linear B-Cell Epitopes Using String Kernels," Journal of Molecule Recognition, Vol. 21, No. 4, 2008, pp. 243-255. doi: $10.1002 / \mathrm{jmr} .893$

[19] S. K. Srivastava, S. Sankar, H. M. Harshan and S. R. Somarajan, "Evaluation of a Recombinant LigB Protein of Leptospira Interrogans Serovar Canicola in an Enzyme-Linked Immunosorbent Assay for the Serodiagnosis of Bovine Leptospirosis," Research in Veterinary Science, Vol. 88, No. 3, 2010, pp. 375-378.

doi:10.1016/j.rvsc.2009.11.004 